\title{
Damping Effects Induced by a Mass Moving along a Pendulum
}

\author{
E. Gandino, S. Marchesiello, A. Bellino, A. Fasana, and L. Garibaldi \\ Department of Mechanical and Aerospace Engineering, Politecnico di Torino, C.so Duca degli Abruzzi 24, 10129 Torino, Italy
}

Correspondence should be addressed to S. Marchesiello; stefano.marchesiello@polito.it

Received 9 July 2013; Accepted 19 December 2013; Published 1 June 2014

Academic Editor: Nuno Maia

Copyright (C) 2014 E. Gandino et al. This is an open access article distributed under the Creative Commons Attribution License, which permits unrestricted use, distribution, and reproduction in any medium, provided the original work is properly cited.

\begin{abstract}
The experimental study of damping in a time-varying inertia pendulum is presented. The system consists of a disk travelling along an oscillating pendulum: large swinging angles are reached, so that its equation of motion is not only time-varying but also nonlinear. Signals are acquired from a rotary sensor, but some remarks are also proposed as regards signals measured by piezoelectric or capacitive accelerometers. Time-varying inertia due to the relative motion of the mass is associated with the Coriolis-type effects appearing in the system, which can reduce and also amplify the oscillations. The analytical model of the pendulum is introduced and an equivalent damping ratio is estimated by applying energy considerations. An accurate model is obtained by updating the viscous damping coefficient in accordance with the experimental data. The system is analysed through the application of a subspace-based technique devoted to the identification of linear time-varying systems: the so-called short-time stochastic subspace identification (ST-SSI). This is a very simple method recently adopted for estimating the instantaneous frequencies of a system. In this paper, the ST-SSI method is demonstrated to be capable of accurately estimating damping ratios, even in the challenging cases when damping may turn to negative due to the Coriolis-type effects, thus causing amplifications of the system response.
\end{abstract}

\section{Introduction}

The analysis and simulation [1] of mechanical systems with imposed relative motion of components are challenging: time-varying inertia, created by a mass sliding along a rotating member, is associated with Coriolis-type effects. The relative movement can excite or reduce the structure vibration, providing new means or techniques for active amplification or attenuation of vibrations. A variable length mathematical pendulum was used in [2] to examine the concept of controlling the motion of a system through mass reconfiguration, that is, by sliding a mass towards and away from the pivot. A variable length pendulum has also been considered in [3], where a rigorous qualitative investigation of its equation is carried out without any assumption on small swinging amplitudes. In [4] a physical pendulum was considered to present a technique in which a radially moving mass is treated as a controller to attenuate the pendulum swings. A moving mass is a proper characteristic of a timevarying system, which is in general one of the sources of nonstationary signals. Another source can be, in case of a pendulum, nonlinearity due to its large swinging amplitudes. The oscillations are also associated with the effective damping ratio, which is explicitly determined in [4] from energy considerations in terms of the mass motion pattern and the pendulum parameters. This is only one of the several techniques that can be adopted to estimate the damping ratios of a system and in fact it can be seen as part of the larger problem of dynamic identification, as it is actually proposed in this paper.

During the last years, many efforts have been spent in studying nonstationary signals. Among the first works on the identification of time-varying systems, $[5,6]$ introduced the concept of pseudonatural frequencies that are obtained by the time-varying state transition matrix. The work in [7] proposed a recursive algorithm, based on subspace methods, to identify the state matrices and consequently to determine the modal parameters. Other important approaches are those based on the Kalman Filter [8], or the parametric methods as, for example, the FS-TARMA [9], which is an extension of the classical ARMA techniques. In [10], a Short-Time Stochastic Subspace Identification (ST-SSI) approach has been defined, based on the "frozen" technique, where the classical subspace identification [11] is applied to a sequence of windowed parts of the signal. 
The ST-SSI method can be applied to different kinds of nonstationary systems in order to estimate the instantaneous frequencies and, for example, it has been used to estimate the frequency in practical systems showing nonlinear effects [12]. However, instead of extracting a series of time-varying linear models, the identification of a whole parametric nonlinear model is an important instrument for many purposes. Among the past and recent developments [13], the nonlinear subspace identification (NSI) method has been developed in [14] and improved in [15] for identifying large systems with lumped nonlinearities. Both the ST-SSI and the NSI methods have been applied in [16] for estimating the swinging frequency of an experimental time-varying inertia pendulum, whose dynamics is governed by a nonlinear equation of motion due to large swinging amplitudes.

The same pendulum is considered in this paper for investigating a suitable model of damping. The paper starts with the description of the experimental set-up. Signals are acquired from a rotary sensor, but some remarks are also proposed for signals measured by piezoelectric or capacitive accelerometers. After the introduction of the analytical equation of motion, the energy approach of [4] and the ST-SSI method are briefly described and applied for estimating the equivalent damping ratio. When the mass is fixed, the energy approach is employed for updating the preliminary model in order to fit the experimental data. Three moving-mass cases are finally analysed to demonstrate that the estimates of the damping factors obtained by means of ST-SSI are very accurate, when compared with those obtained by an analytical model and by the energy approach.

\section{Experimental Set-Up}

The structure under test is a pendulum with time-varying inertia: a disk on a cart can slide along a runner, while the pendulum is swinging. This structure cannot be considered simply as a linear time-variant system, the equation of motion of the pendulum being nonlinear for large swinging amplitudes.

2.1. Description. An overview of the design of the structure is presented in this section, together with a description of the instrumentation used for data acquisition. Further details about the experimental set up and the measured characteristics of the considered elements can be found in [16]. The pendulum is formed by a thin aluminium runner allowing the sliding of a cart which can host an added mass: the motion of the mass varies the pendulum inertia. Moreover, in order to avoid a nonoptimal clamp between the runner and the shaft due to the large deformability of aluminium, a small metallic plate has also been added at the root of the aluminium beam, near the hinge, to limit its transverse vibrations.

The travelling mass is a steel disk of mass $m_{m}=$ $0.5025 \mathrm{~kg}$, whose motion is regulated by a hand-driven counterbalancing mass. The latter is connected to the moving mass through a system of pulleys and a cable that can be considered as nonextendible. The complete structure is shown in Figure 1(a): the main supports, plates, pulleys, bearings, and precision shaft are visible.

The sensors can also be seen in Figure 1(a). A triaxial and four monoaxial accelerometers have been mounted along the beam. The triaxial accelerometer, a PCB $356 \mathrm{~B} 18$ piezoelectric sensor (ICP), is used to express some practical considerations in Section 2.2. To show typical measurement errors, some data sets have been acquired by adding a capacitive accelerometer to the system (not shown in the figure), in the same position of the ICP sensor. Each monoaxial accelerometer is a Brüel \& Kjær 4507 B 004 piezoelectric sensor, used to measure the transversal vibrations (along the $z$ axis in Figure 1(b)) of the pendulum [16]. Their signals have not been analysed in this paper.

A direct measure of the angular position of the pendulum is given by a Penny+Giles SRS280 sealed rotary sensor, with an accuracy of $\pm 1 \%$ over $100^{\circ}$, connected to the precision shaft. A Celesco PT1A linear potentiometer, with a maximum extension of $1.2 \mathrm{~m}$, has been connected to the counterweight (see Figure 1(a)). The position of the travelling mass along the runner can be simply obtained from this measure.

All signals have been acquired and recorded with a sampling frequency of $256 \mathrm{~Hz}$ by using an OROS acquisition system, with 32 channels and antialiasing filter.

2.2. Remarks about the Accelerometers. In this section some remarks about the signals measured by the accelerometers are proposed, together with a comparison with the rotary sensor recordings. In the following, $\theta(t)$ is the output of the rotary potentiometer, which is very accurate at these low frequencies; thus the use of supplementary sensors is not needed to describe the dynamics of the SDOF system. However, accelerometers are mounted to give some useful guidelines in case a potentiometer is not available. A piezoelectric sensor (ICP) is not suited to measure the radial acceleration of the pendulum under exam, because it removes the DC component of the output, which is nonnull. This is why a capacitive accelerometer was chosen for comparison.

To show the difference, the two accelerometers have been mounted on the beam in the same position $s_{a}=0.93 \mathrm{~m}$, with radial direction (the $x$ axis in Figure 1(b)). The signal $\theta(t)$ of the rotary sensor has been numerically differentiated in order to obtain $\dot{\theta}(t)$, which is used for computing the "actual" value of the radial acceleration $a_{r}(t)=s_{a} \dot{\theta}(t)^{2}$, shown in Figure 2(a). The signals acquired by the accelerometers are represented in Figure 2(b): the ICP measurement $\tilde{a}_{r, \text { ICP }}(t)$ has zero mean and its value is zero for $\theta=0$ and $\dot{\theta}=0$ (at the end of time history), while the capacitive sensor output $\tilde{a}_{r, \text { cap }}(t)$ is asymmetric and its value tends to $g$ for $\theta=0$ and $\dot{\theta}=0$. Clearly, none of the two behaviours can be associated with the actual value of radial acceleration.

Another remark arises from Figure 2(b): the effect of the gravitational acceleration $g$ on the measured signals must be taken into account and removed in order to get the correct value of the radial acceleration. This is due to the fact that the measurement axes of the accelerometer on the pendulum have an orientation that largely changes over time, while most of dynamics applications do not show such a 


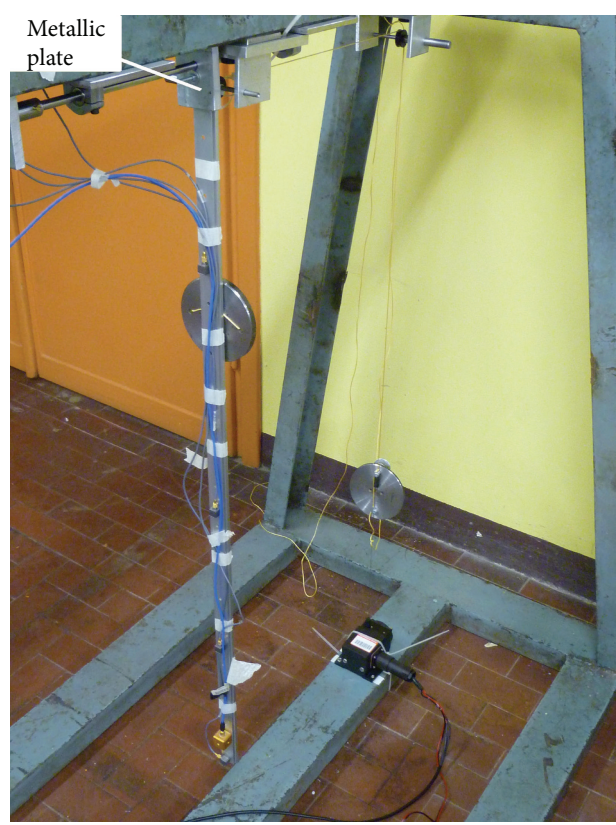

(a)

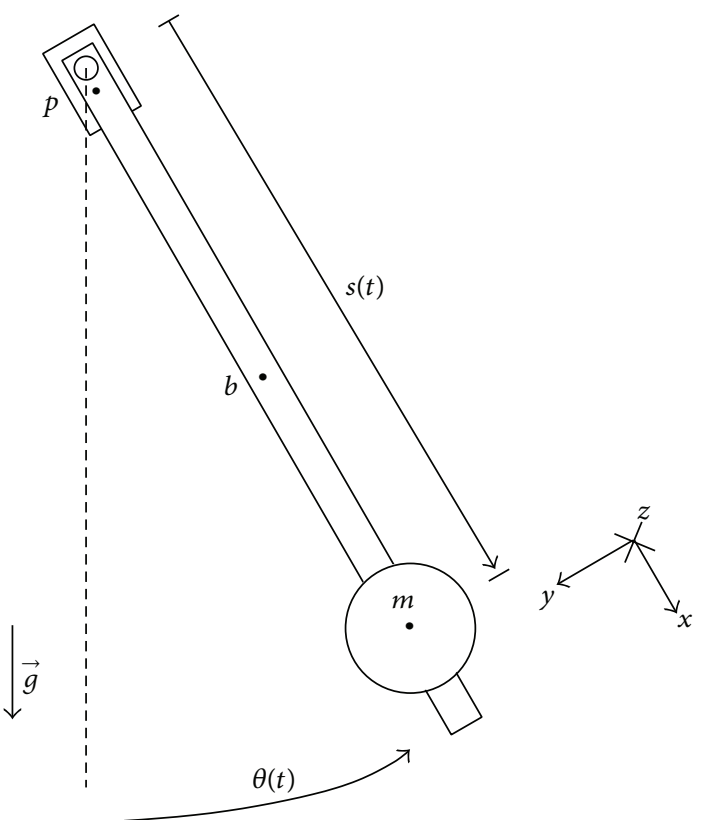

(b)

Figure 1: (a) Complete structure. At the top, the reinforcement plate is highlighted. The travelling mass and the counterweight are visible on the left and on the right, respectively. (b) Pendulum with the travelling mass.

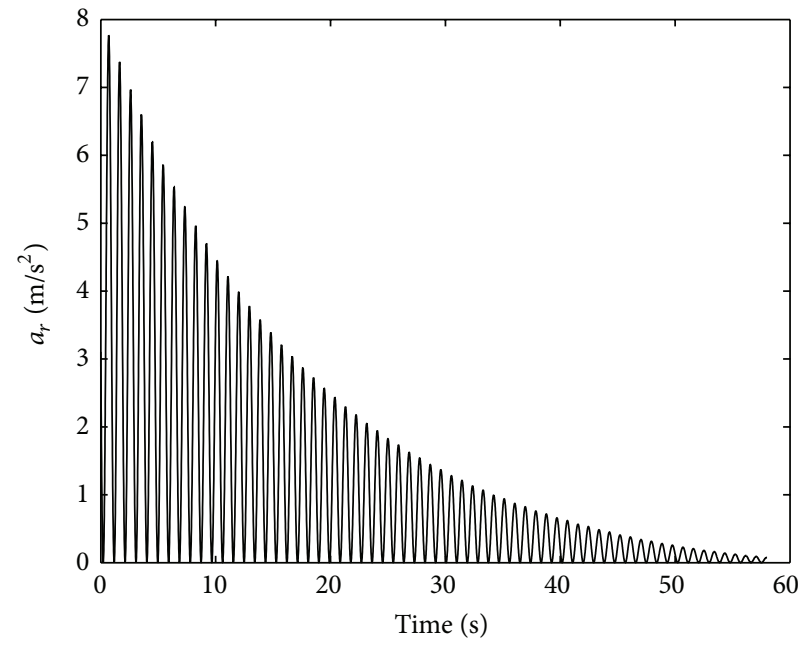

(a)

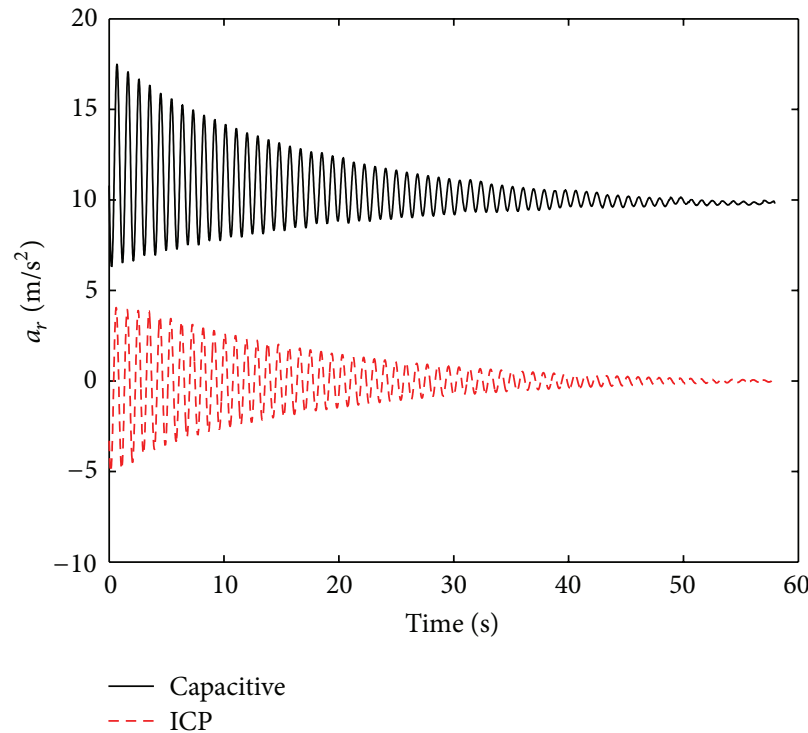

(b)

Figure 2: (a) Actual value of the radial acceleration. (b) Measured radial accelerations, by the capacitive and the ICP sensor.

behaviour. A "cleaning" operation can be thought of as being trivial, but it cannot be performed on signals from classical ICP accelerometers, because of the continuous component removal described above. When the capacitive sensor signal is considered as "corrupted" by the presence of $g$, an estimate (indicated by $\wedge$ ) of the actual radial acceleration is obtained after cleaning:

$$
\tilde{a}_{r, \text { cap }}(t)=a_{r}(t)+g \cos \theta(t)
$$

$$
\stackrel{\text { CLEANING }}{\longrightarrow} \widehat{a}_{r, \text { cap }}(t)=\widetilde{a}_{r, \text { cap }}(t)-g \cos \theta(t) .
$$

Figure 3(a) shows a comparison between the actual radial acceleration $a_{r}(t)$ and the estimate (1b): an almost perfect correspondence is now obtained. A similar approach can be adopted if the tangential direction (the $y$ axis in Figure 1(b)) is considered. By differentiating again the signal $\dot{\theta}(t)$ in order to obtain $\ddot{\theta}(t)$, the "actual" value of the tangential acceleration 


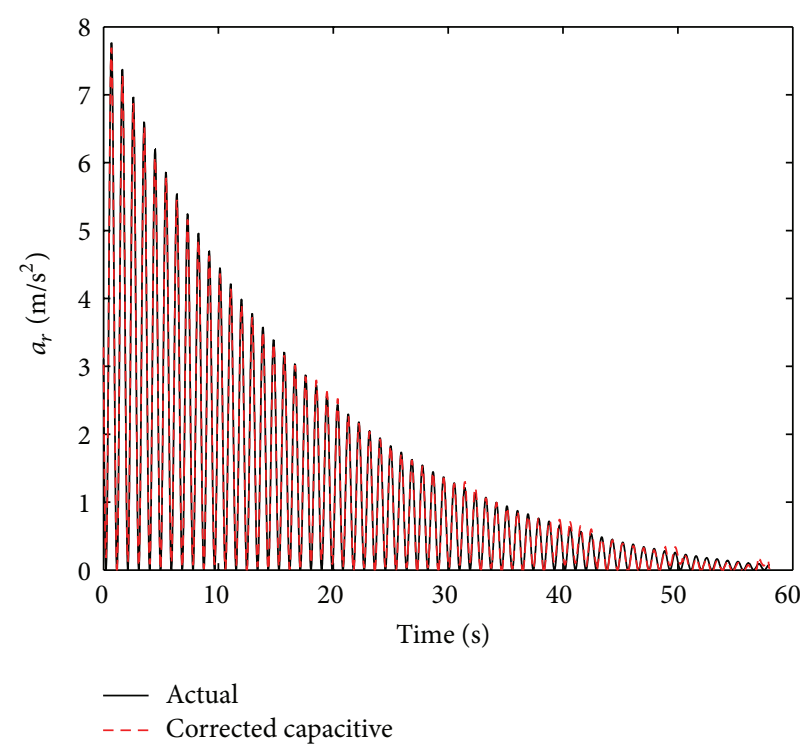

(a) Radial direction

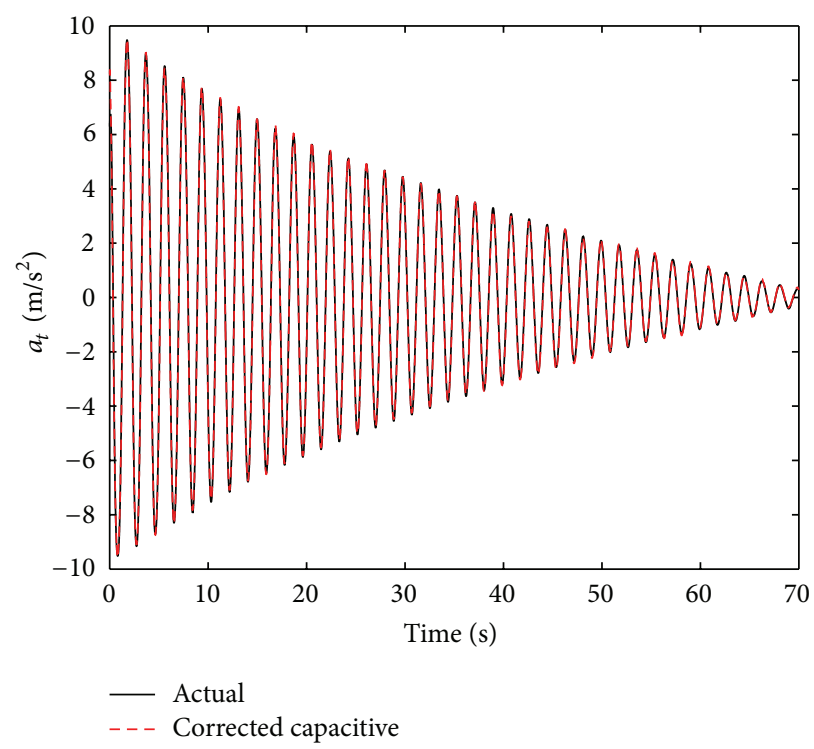

(b) Tangential direction

FIGURE 3: Comparisons between actual accelerations and corrected capacitive estimates.

$a_{t}(t)=s_{a} \ddot{\theta}(t)$ has been computed. The capacitive estimate $\widehat{a}_{t, \text { cap }}(t)$ can be obtained from the measured signal $\widetilde{a}_{t, \text { cap }}(t)$ as $\hat{a}_{t, \text { cap }}(t)=\tilde{a}_{t, \text { cap }}(t)-g \sin \theta(t)$. The comparison between the actual tangential acceleration and the capacitive estimate is shown in Figure 3(b): again, a perfect agreement can be observed. In conclusion, an angle measurement is necessary in this kind of problems.

2.3. Equation of Motion. The system shown in Figure 1(b) consists of a rigid bar with no flexural effects; the pendulum is simply a nonlinear SDOF system.

From the rotational equilibrium of the system, the equation of the swinging motion is

$$
\begin{aligned}
\left(I_{\text {up }}\right. & \left.+m_{m} s(t)^{2}\right) \ddot{\theta}(t) \\
& +\left(c_{v}+2 m_{m} s(t) \dot{s}(t)\right) \dot{\theta}(t)+\left(P_{\text {up }}+g m_{m} s(t)\right) \sin \theta(t) \\
= & I_{\text {tot }}(t) \ddot{\theta}(t)+C_{\text {tot }}(t) \dot{\theta}(t)+P_{\text {tot }}(t) \sin \theta(t)=0,
\end{aligned}
$$

in which the subscript "up" refers to the quantities not depending on the mass position $s(t)$. These have been estimated by means of the Nonlinear Subspace Identification (NSI) method in [16], in which an updating procedure was performed to build an accurate model tuned on the measured results. The angle swept by the pendulum is indicated by $\theta(t)$ whilst $s(t)$ is the distance of the disk from the hinge. Other terms appearing in (2) are the gravitational acceleration $g=$ $9.81 \mathrm{~m} / \mathrm{s}^{2}$ and a viscous damping coefficient $c_{v}$. Given the value $m_{m}=0.5025 \mathrm{~kg}$ of the travelling mass, model (2) can be completely defined by exploiting the results obtained in [16]: $I_{\text {up }}=0.1292 \mathrm{~kg} \mathrm{~m}^{2} ; P_{\text {up }}=1.8380 \mathrm{~kg} \mathrm{~m}^{2} \mathrm{~s}^{-2}$; moreover, an overall estimate of $c_{v}=0.035 \mathrm{~kg} \mathrm{~m}^{2} \mathrm{~s}^{-1}$ is used for preliminary comparisons.
The analytical form of the time-variant viscous damping ratio is then defined as

$$
\zeta(t)=\frac{C_{\text {tot }}(t)}{2 \sqrt{I_{\text {tot }}(t) P_{\text {tot }}(t)}} .
$$

\section{Methodologies}

Two methodologies are adopted in this paper for obtaining estimates of the equivalent viscous damping ratio. The first, based on energy considerations, is evaluated as a baseline for updating the model and is used for comparisons with the second technique, based on subspace identification.

3.1. Energy Considerations. A method for deriving an estimate of the damping ratio, which is briefly described in this section and used for successive comparisons, can be found in [4]. The normalised total energy of the pendulum with moving mass can be written as

$$
E(t)=\frac{1}{2} \dot{\theta}(t)^{2}+\omega_{s}(t)^{2}(1-\cos \theta(t))
$$

where $\omega_{s}(t)^{2}=\left(P_{\text {up }}+g m_{m} s(t)\right) /\left(I_{\text {up }}+m_{m} s(t)^{2}\right)$ is related to the "instantaneous" frequency (when mass $m_{m}$ is at location $s(t)$ ). The two terms of $E(t)$ can be interpreted as the normalised kinetic and potential energies of the pendulum, respectively. In [4] a mathematically equivalent form of (2) is rewritten, involving $E(t)$ and its rate of change; then, an integral form is considered over one swinging period $T$ and the following approximation of the damping ratio for the pendulum with moving mass is defined:

$$
\zeta \cong \frac{1}{4 \pi} \frac{E(0)-E(T)}{E(0)} .
$$


Thus, the damping ratio is defined in (5) by the normalised energies that can always be calculated for a given set of conditions at the beginning (represented by $E(0)$ ) and at the end $(E(T))$ of each cycle. In this way, a single value for $\zeta$ can be obtained at each cycle, in order to get an approximation of the "instantaneous" damping ratio expressed by (3). This method has two main limitations: (i) every estimate of $\zeta$ from (5) can be obtained only if exactly a cycle is considered; (ii) equation (5) involves the application of (4), which implies full knowledge of the pendulum parameters and the mass motion pattern, contained in the definition of $\omega_{s}(t)$.

3.2. Subspace Identification. The procedure for the identification of linear time-varying systems is called Short-Time Stochastic Subspace Identification (ST-SSI) [10, 17]. The basic idea of the method consists of windowing the signal into many parts and considering the system as time-invariant in each time window: the process is called frozen technique.

If the output data are measured at discrete times with a sampling interval $\Delta t$ and the input is a discrete signal characterised by a zero-order hold between consecutive sample points, the discrete-time state-space model of a general linear time-varying system at a time instant $t=r \Delta t$ can be obtained. The frozen technique considers constant state matrices during each time step, so that the following representation can be adopted:

$$
\begin{aligned}
& \left\{\begin{array}{l}
x(r+1)=A(r) x(r)+B(r) u(r)+w(r) \\
y(r)=C(r) x(r)+D(r) u(r)+v(r)
\end{array}\right. \\
& \stackrel{\text { FROZEN TECHNIQUE }}{\longrightarrow}\left\{\begin{array}{l}
x(r+1)=A x(r)+B u(r)+w(r) \\
y(r)=C x(r)+D u(r)+v(r),
\end{array}\right.
\end{aligned}
$$

where $A(r)$ and $B(r)$ are not constant and in general their closed forms are unknown $[6] ; x(r)$ is the state vector, $u(r)$ the input vector, and $y(r)$ the output vector; $w(r)$ and $v(r)$ are process and measurement error, respectively. The complete time record is split into time windows (frozen system), whose length corresponds to a period $T$ (about 400 samples) for comparing the ST-SSI results with those obtained through the energy approach of previous Section 3.1: such a restriction to a cycle, however, is not a limitation of the present ST-SSI method. Usually, the windows are almost completely overlapped except for a sampling period $\Delta t$ (or a multiple) and their length can be arbitrarily chosen. If window lengths are short, the data-driven subspace method [11] is preferred with respect to the covariance-driven version [18], which needs more samples to obtain accurate results. Subspace methods do not need any a priori knowledge of the system parameters, as they identify the state-space matrices of (6b) starting from the measured system responses. Natural frequencies and damping ratios are then extracted by computing the eigenvalues of the identified matrix $A$ in every window. An extensive study about the time-varying swinging frequency of the pendulum has been performed in [16], in which the contribution of nonlinearity was analysed by means of the Nonlinear Subspace Identification (NSI) method [14]. This paper is focused on the more challenging topic of damping.

\section{Results}

In this section the results are presented. At first, the mass is fixed and preliminary considerations about the model are drawn: the overall estimate of the viscous damping coefficient is updated to fit the experimental results and an accurate model is thus obtained. Then, three moving-mass cases are considered and the results obtained by the ST-SSI method are validated by comparisons with the method in [4] and the updated analytical model.

4.1. Fixed Mass. By considering the mass fixed in $s(t)=$ $\bar{s}=49 \mathrm{~cm}$ (and obviously $\dot{s}(t) \equiv 0$ ), (5) can be applied and the obtained damping factors can be used to compute estimates for the viscous damping coefficient, at each cycle. These values can be then compared with the overall estimate $c_{v}=0.035 \mathrm{~kg} \mathrm{~m}^{2} \mathrm{~s}^{-1}$, defined by a preliminary nonlinear identification [16]. The comparison is shown in Figure 4(a): it is clear that a constant $c_{v}$ value is not proper for representing the experimental evidence. An updating procedure is then carried out for obtaining a new model in which the viscous damping coefficient is no more constant: indeed it is a function of the root mean square (RMS) of angular velocity in each cycle, named $\tilde{\dot{\theta}}$. The updating simply consists of a polynomial fitting of the viscous coefficients obtained through the energy considerations of Section 3.1. In particular, the variable $C_{\text {tot }}$ is updated to $\widetilde{C}_{\text {tot }}=c_{v}(\tilde{\dot{\theta}})+2 m_{m} s(t) \dot{s}(t)$ and, consequently, the analytical form (3) of the viscous damping ratio changes into the following "updated" version:

$$
\zeta_{\text {up }}(t)=\frac{\widetilde{C}_{\text {tot }}(t)}{2 \sqrt{I_{\text {tot }}(t) P_{\text {tot }}(t)}} .
$$

Figure 4(a) shows the behaviour of $\tilde{c}_{v}$ during a swinging decay: as expected, it accurately fits the energy approach estimates. In Section 4.2, the new damping model (7) will be the baseline for comparisons in more complicated situations involving the mass moving along the beam.

In order to have a better visualisation of the experimental damping characteristic, the damping force $F_{v}=c_{v}(\tilde{\dot{\theta}}) \tilde{\dot{\theta}}$ is represented in Figure 4(b) as a function of $\tilde{\dot{\theta}}$. Two zones can be distinguished: for small angular velocities $(\tilde{\dot{\theta}}<$ $0.05 \mathrm{rad} \mathrm{s}^{-1}$ ) a Coulomb friction is prevalent, while for larger angular velocities the contribution of the viscous damping is more evident.

4.2. Moving Mass. In this section three moving-mass cases are considered, in order to show the results obtained by means of the ST-SSI method. In Figure 5 the measured mass positions along the beam and the swinging amplitudes over time are shown, for each case.

4.2.1. Case 1. The mass is moving downwards, from the top to the middle of the beam (Figure 5(a)). No evidence of increasing swinging amplitudes due to the Coriolis-type effect can be observed in Figure 5(b), since the velocity $\dot{s}$ is positive. 


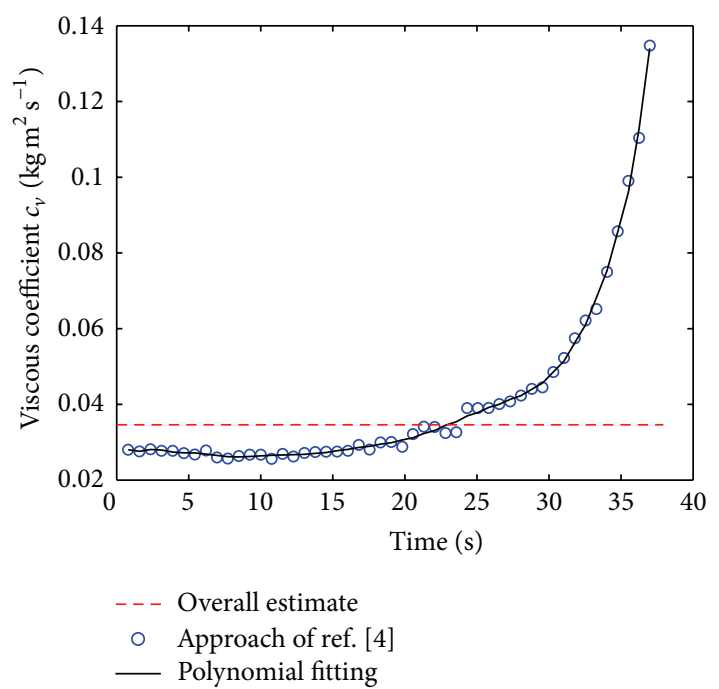

(a)

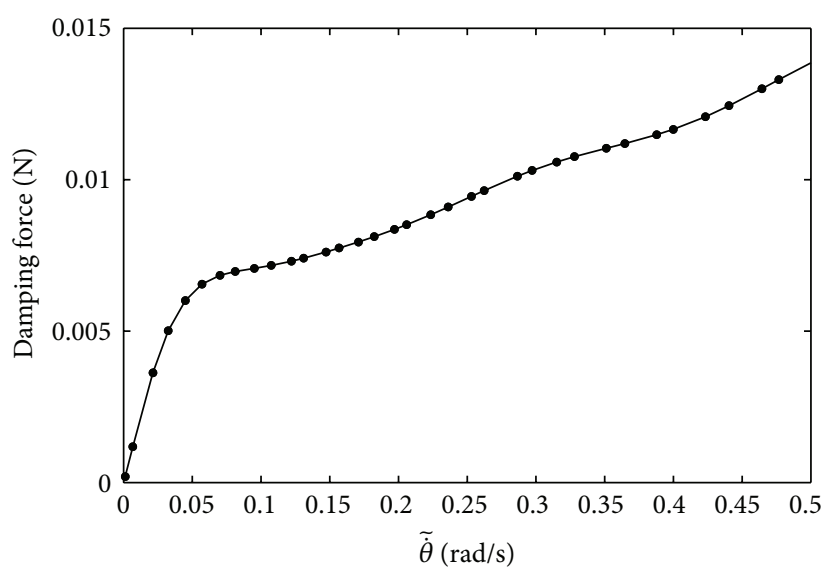

(b)

Figure 4: Fixed-mass case. (a) Viscous coefficient as a function of time, during a swinging decay. (b) Damping force as a function of $\tilde{\dot{\theta}}$ at each cycle.

Case 1 is useful for demonstrating once again the need for the updating procedure performed in Section 4.1. Estimates of the damping factors, obtained through the energy approach of Section 3.1 and the ST-SSI method of Section 3.2, are compared with the overall estimate of (3) in Figure 6(a): the energy-approach and the ST-SSI estimates are similar, but there is no correspondence with the overall estimate. The same estimates can be compared with the updated value of (7) in Figure 6(b): a good level of agreement can be observed, confirming the reliability of the damping model obtained in Section 4.1 for a fixed mass. As a general remark emerging from Figure 6, the ST-SSI method gives slightly overestimated values of damping, with respect to those obtained by the energy approach. The reason is the model order $n$ selected for representing the state-space model (6b): in this paper it has been fixed to 2 for simplicity, but more accurate estimates of damping can be obtained by increasing $n$ and by investigating the results by means of stabilisation diagrams.

4.2.2. Case 2. The mass is moving downwards and upwards, in an almost regular way (Figure 5(c)). An excitation of the swinging amplitudes due to the Coriolis-type effect can be observed in Figure 5(d) at about 10 seconds, since the velocity $\dot{s}$ is negative and large enough to change the sign of $\zeta_{\text {up }}$ in (7). A less clear excitation can also be observed after 20 seconds. These qualitative considerations are confirmed by the damping factors represented in Figure 7(a). The energyapproach and the ST-SSI estimates are compared with the updated value of (7): an excellent agreement can be observed for the ST-SSI estimates.

4.2.3. Case 3. This is the more challenging case: the mass is moving along the beam, in a more irregular way especially from 5 to 12 seconds (Figure 5(e)). From a detailed inspection of Figure 5(f), four zones are recognised, in which an amplification of the swinging amplitudes due to the Coriolis-type effect can be observed: they are marked by letters A, B, C, and $\mathrm{D}$. In particular, zones $\mathrm{A}$ and $\mathrm{B}$ are short and could be difficult to distinguish, but the representation of the damping factors in Figure 7(b) can be useful in this sense. In fact, Figure 7 (b) reveals the four zones in which the equivalent damping factor is negative and these are found to correspond to those of Figure 5(f). Moreover, in Figure 7(b) the energyapproach and the ST-SSI estimates are compared with the updated value of (7): the ST-SSI estimates are very accurate.

As a final consideration, observe that Figures $7(\mathrm{a})$ and 7(b) are represented on the same scale: Case 3 appears to be more challenging also because larger and fast-varying values of damping factors are involved. However, results are satisfactory even in such a complicated case, in which high mass velocities cause substantial changes in the system dynamics.

\section{Conclusions}

The experimental study of damping in a time-varying inertia pendulum is presented. The system consists of a disk travelling along a pendulum: this relative motion, which is associated with Coriolis-type effects, can be exploited for attenuation or amplification of the pendulum oscillations. At first, signals measured by piezoelectric or capacitive accelerometers are compared with the output of a rotary sensor: for swinging systems like the pendulum under exam, the piezoelectric sensor is not suited because it removes the DC component of the output. The effect of gravitational acceleration can be taken into account and correctly removed from the measured signal of a capacitive acceleration, but angle measurement is needed. 


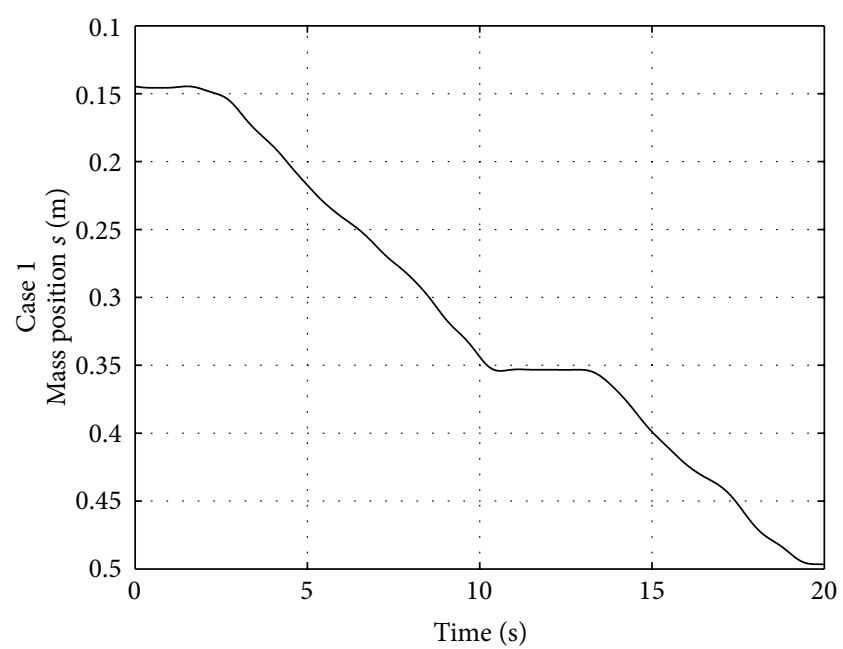

(a)

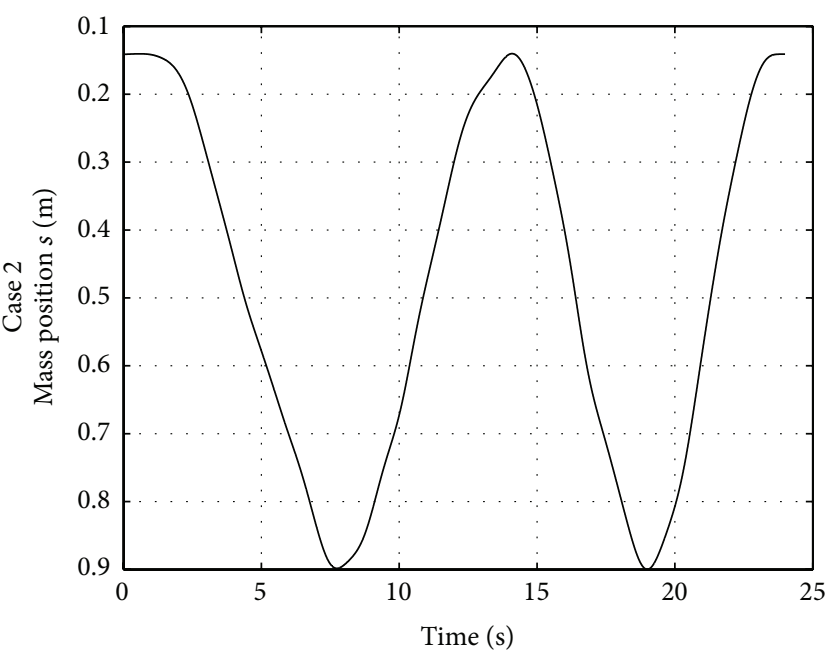

(c)

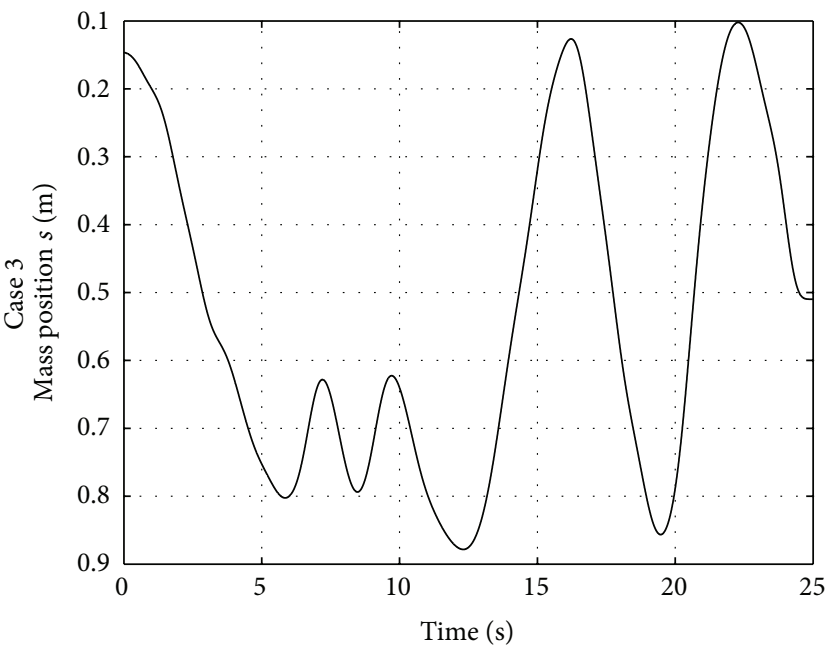

(e)

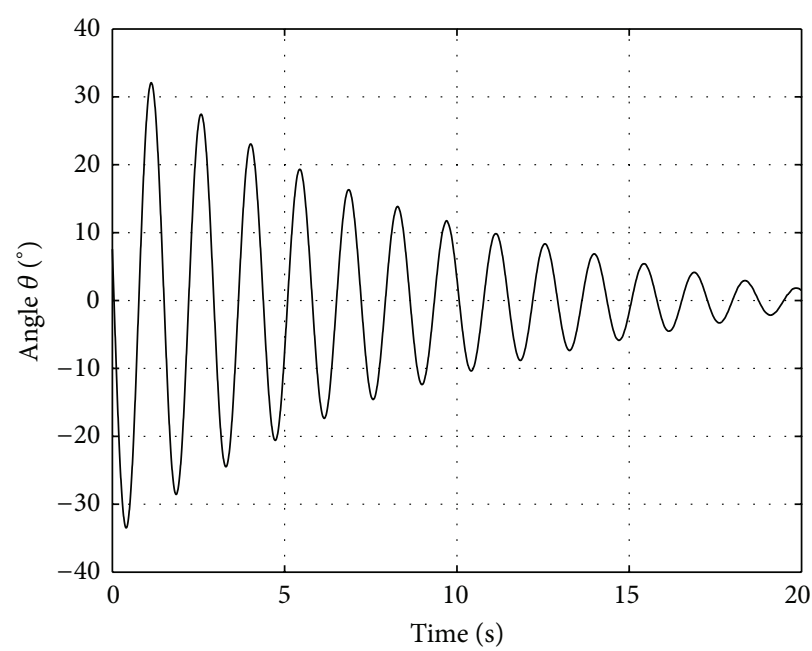

(b)

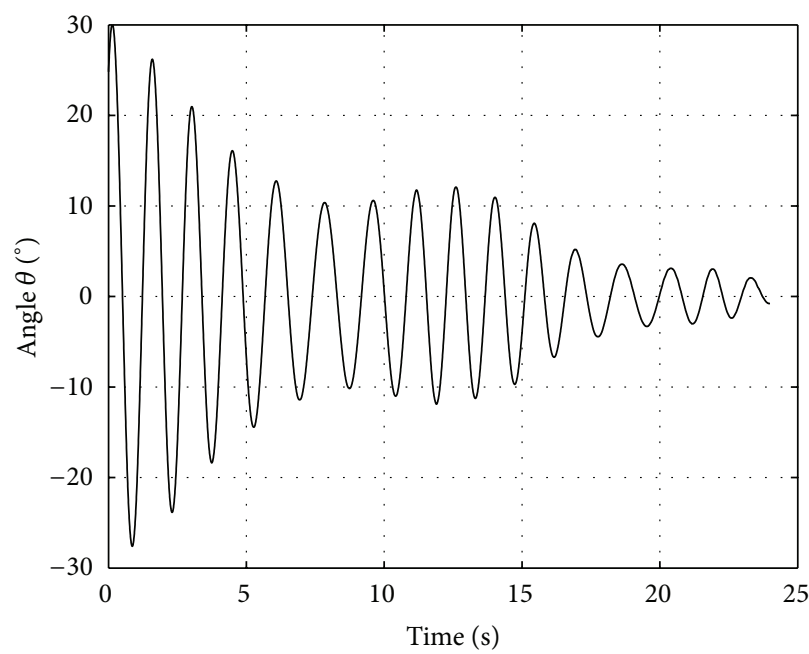

(d)

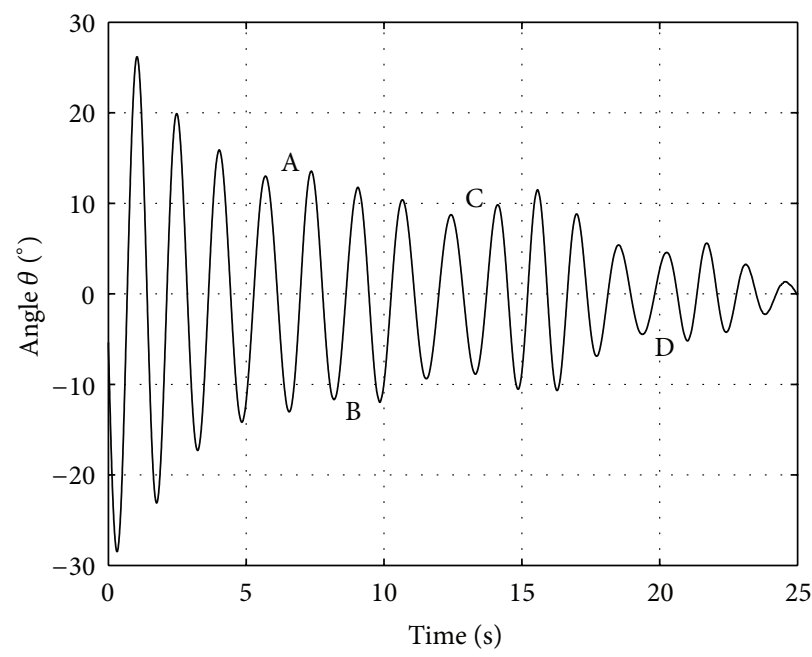

(f)

Figure 5: Moving mass. (a, b) Case 1. (c, d) Case 2. (e, f) Case 3. (a, c, e) Mass position along the beam. (b, d, f) Swinging amplitude over time. 


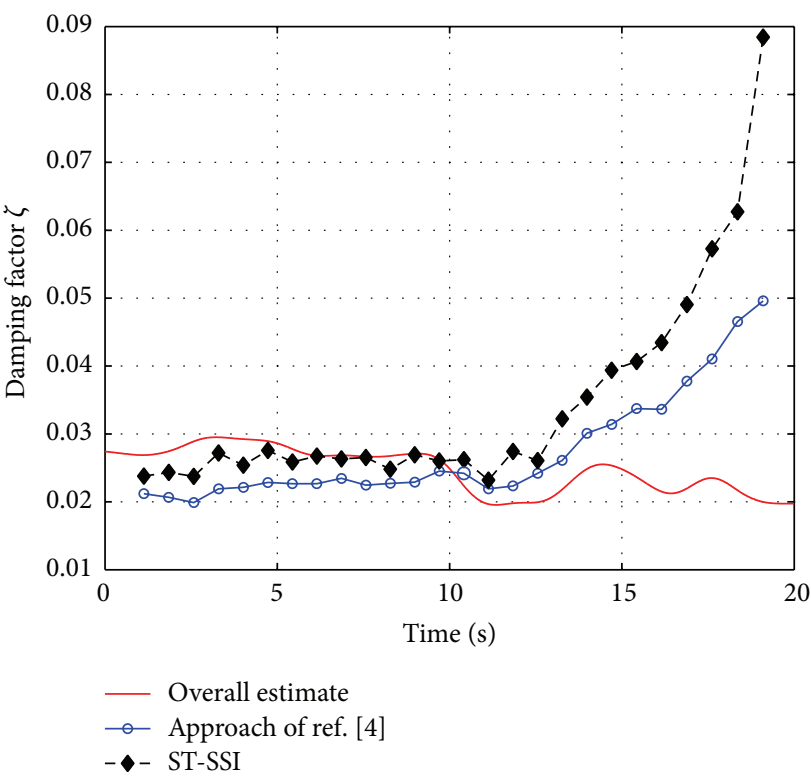

(a)

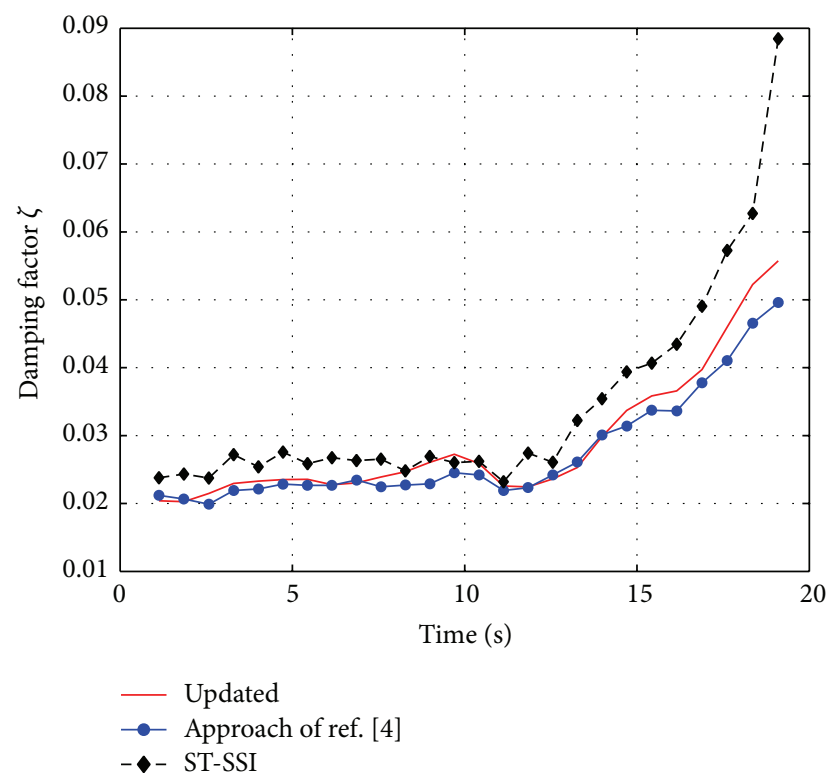

(b)

FIGURE 6: Moving-mass Case 1. Estimates of the damping factors, compared with the overall estimate of (3) (a) and with the updated value of (7) (b).

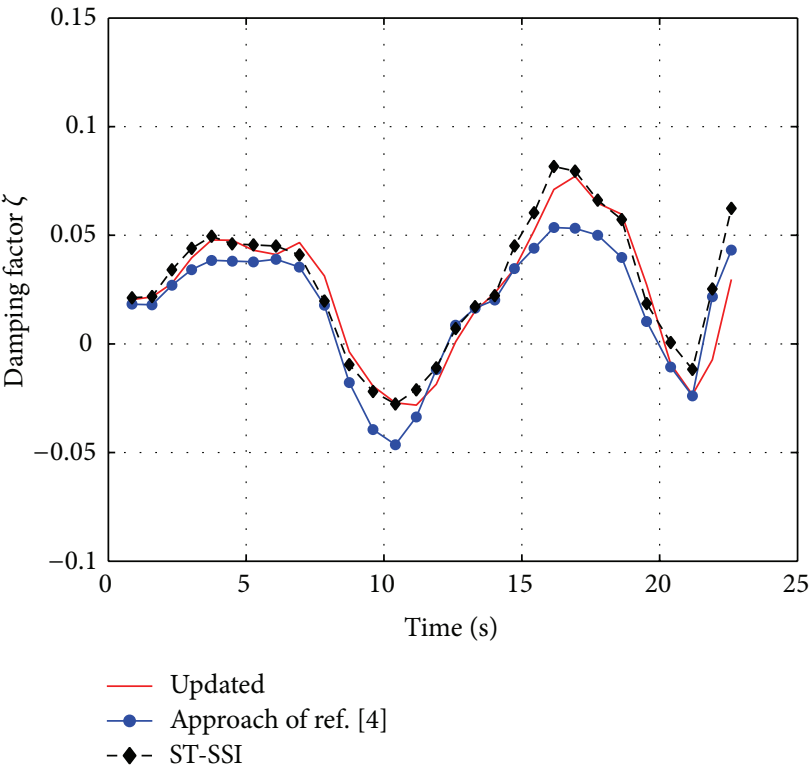

(a)

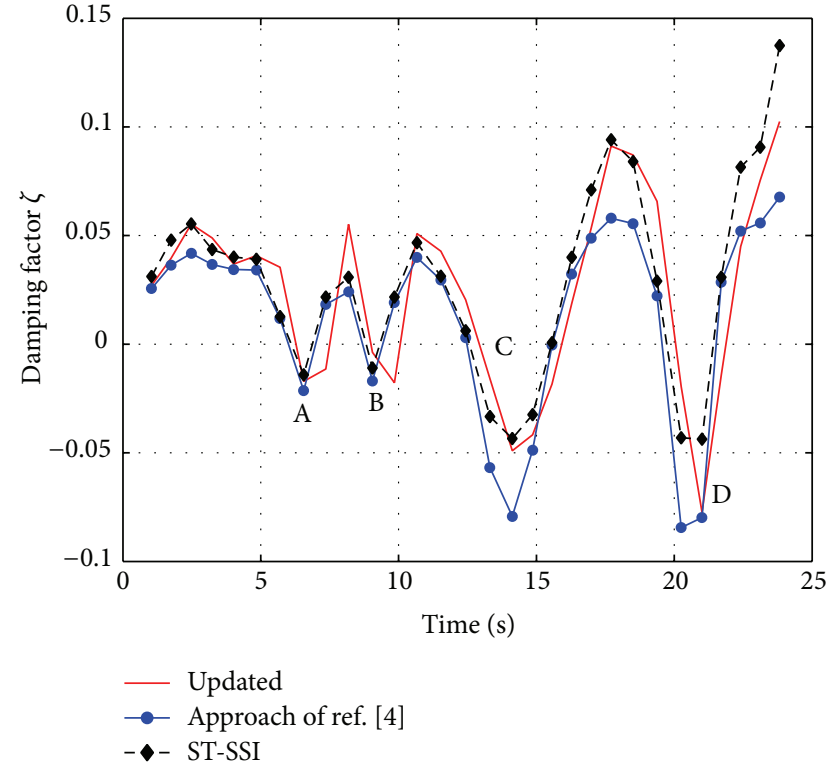

(b)

FIGURE 7: Estimates of the damping factors, compared with the updated value of (7). Moving-mass Case 2 (a) and Case 3 (b).

The analytical equation of motion is then introduced and the theoretical viscous damping coefficient is updated in order to obtain an accurate damping model. The updating procedure investigates the approach of a published work in which the damping ratios are derived from energy considerations, provided that the system parameters and the mass motion pattern are known. The system is analysed through the application of the Short-Time Stochastic Subspace Identification (ST-SSI), which is very simple and does not require any a priori knowledge about the system. The STSSI estimates of the damping factors are compared with those obtained by an analytical model and by the energy approach. Three moving-mass cases are presented, to demonstrate that the ST-SSI estimates are very accurate even in the challenging 
cases in which damping may turn to negative due to the Coriolis-type effects.

\section{Conflict of Interests}

The authors declare that there is no conflict of interests regarding the publication of this paper.

\section{References}

[1] W. Szyszkowski and E. Sharbati, "On the FEM modeling of mechanical systems controlled by relative motion of a member: a pendulum-mass interaction test case," Finite Elements in Analysis and Design, vol. 45, no. 10, pp. 730-742, 2009.

[2] W. Szyszkowski and D. S. D. Stilling, "Controlling angular oscillations through mass reconfiguration: a variable length pendulum case," International Journal of Non-Linear Mechanics, vol. 37, no. 1, pp. 89-99, 2002.

[3] A. A. Zevin and L. A. Filonenko, "A qualitative investigation of the oscillations of a pendulum with a periodically varying length and a mathematical model of a swing," Journal of Applied Mathematics and Mechanics, vol. 71, no. 6, pp. 892-904, 2007.

[4] W. Szyszkowski and D. S. D. Stilling, "On damping properties of a frictionless physical pendulum with a moving mass," International Journal of Non-Linear Mechanics, vol. 40, no. 5, pp. 669-681, 2005.

[5] K. Liu, "Identification of linear time-varying systems," Journal of Sound and Vibration, vol. 206, no. 4, pp. 487-505, 1997.

[6] K. Liu, "Extension of modal analysis to linear time-varying systems," Journal of Sound and Vibration, vol. 226, no. 1, pp. 149167, 1999.

[7] F. Tasker, A. Bosse, and S. Fisher, "Real-time modal parameter estimation using subspace methods: applications," Mechanical Systems and Signal Processing, vol. 12, no. 6, pp. 809-823, 1998.

[8] S.-W. Lee, J.-S. Lim, S. J. Baek, and K.-M. Sung, “Time-varying signal frequency estimation by VFF Kalman filtering," Signal Processing, vol. 77, no. 3, pp. 343-347, 1999.

[9] A. G. Poulimenos and S. D. Fassois, "Output-only stochastic identification of a time-varying structure via functional series TARMA models," Mechanical Systems and Signal Processing, vol. 23, no. 4, pp. 1180-1204, 2009.

[10] A. Bellino, L. Garibaldi, and S. Marchesiello, "Time-varying output-only identification of a cracked beam," Key Engineering Materials, vol. 413-414, pp. 643-650, 2009.

[11] P. van Overschee and B. de Moor, Subspace Identification for Linear Systems: Theory, Implementation, Applications, Kluwer Academic Publishers, Boston, Mass, USA, 1996.

[12] A. Bellino, L. Garibaldi, and S. Marchesiello, "Determination of moving load characteristics by output-only identification over the Pescara beams," Journal of Physics: Conference Series, vol. 305, no. 1, Article ID 012079, 2011.

[13] G. Kerschen, K. Worden, A. F. Vakakis, and J.-C. Golinval, "Past, present and future of nonlinear system identification in structural dynamics," Mechanical Systems and Signal Processing, vol. 20, no. 3, pp. 505-592, 2006.

[14] S. Marchesiello and L. Garibaldi, "A time domain approach for identifying nonlinear vibrating structures by subspace methods," Mechanical Systems and Signal Processing, vol. 22, no. 1, pp. 81-101, 2008.

[15] E. Gandino and S. Marchesiello, "Identification of a duffing oscillator under different types of excitation," Mathematical
Problems in Engineering, vol. 2010, Article ID 695025, 15 pages, 2010.

[16] A. Bellino, A. Fasana, E. Gandino, L. Garibaldi, and S. Marchesiello, "A time-varying inertia pendulum: analytical modelling and experimental identification," Mechanical Systems and Signal Processing, vol. 47, no. 1-2, pp. 120-138, 2014.

[17] S. Marchesiello, S. Bedaoui, L. Garibaldi, and P. Argoul, “Timedependent identification of a bridge-like structure with crossing loads," Mechanical Systems and Signal Processing, vol. 23, no. 6, pp. 2019-2028, 2009.

[18] E. Gandino, L. Garibaldi, and S. Marchesiello, "Covariancedriven subspace identification: a complete input-output approach," Journal of Sound and Vibration, vol. 332, no. 26, pp. 7000-7017, 2013. 

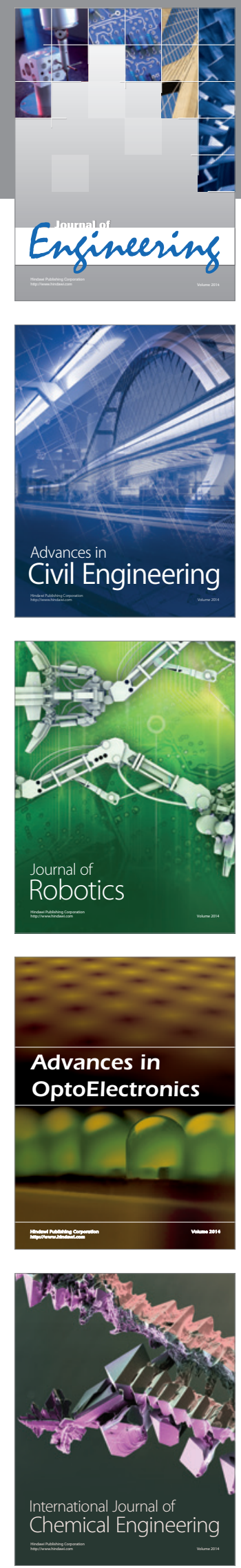

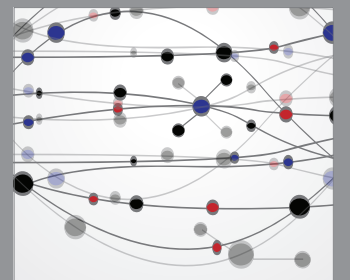

The Scientific World Journal
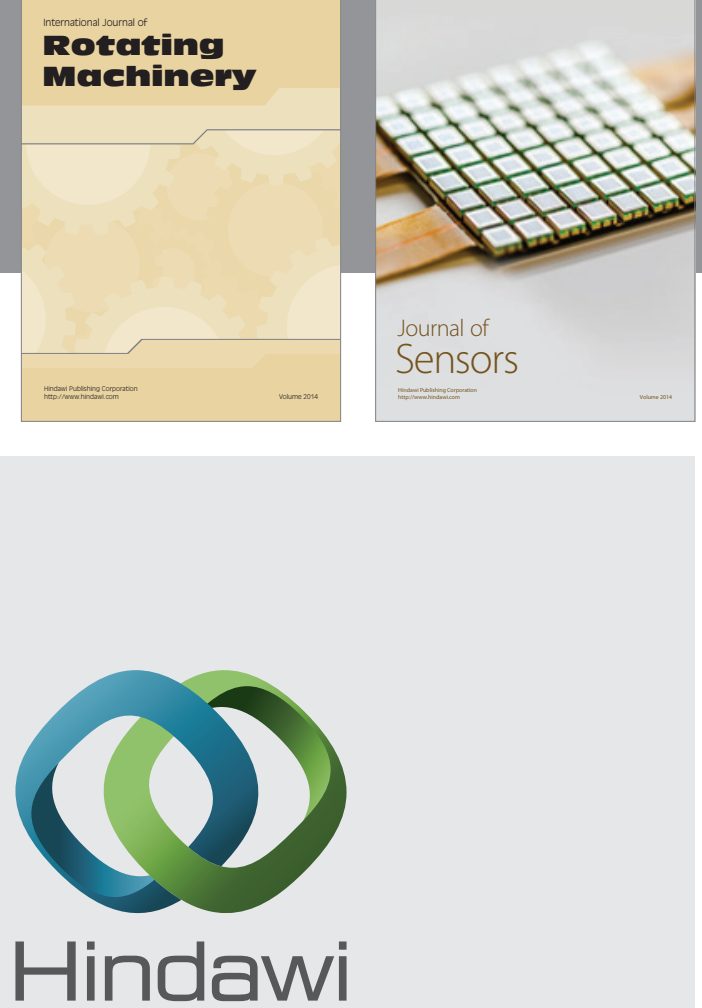

Submit your manuscripts at http://www.hindawi.com
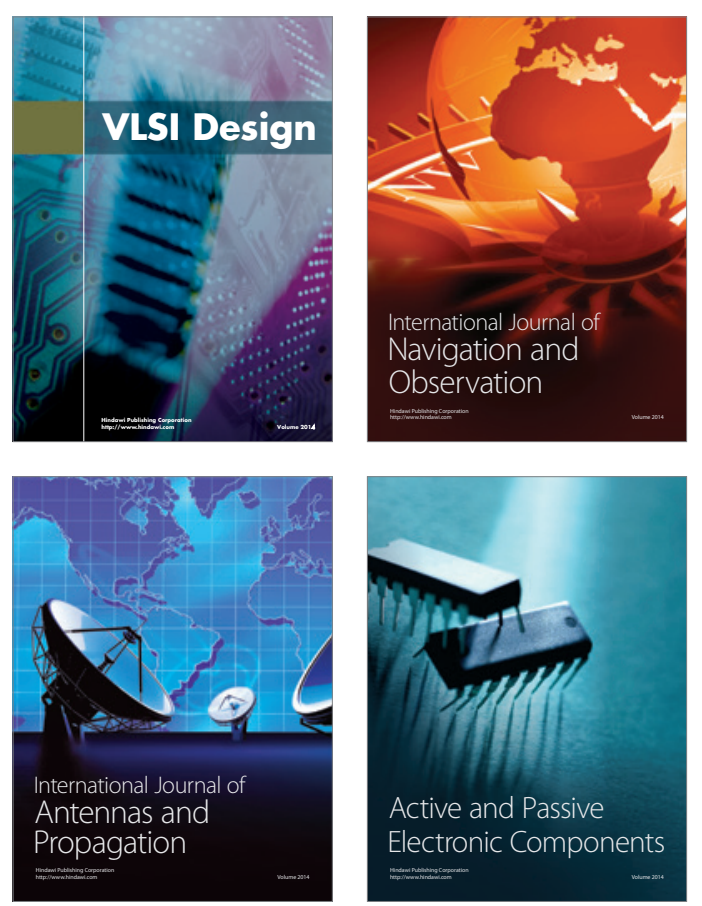
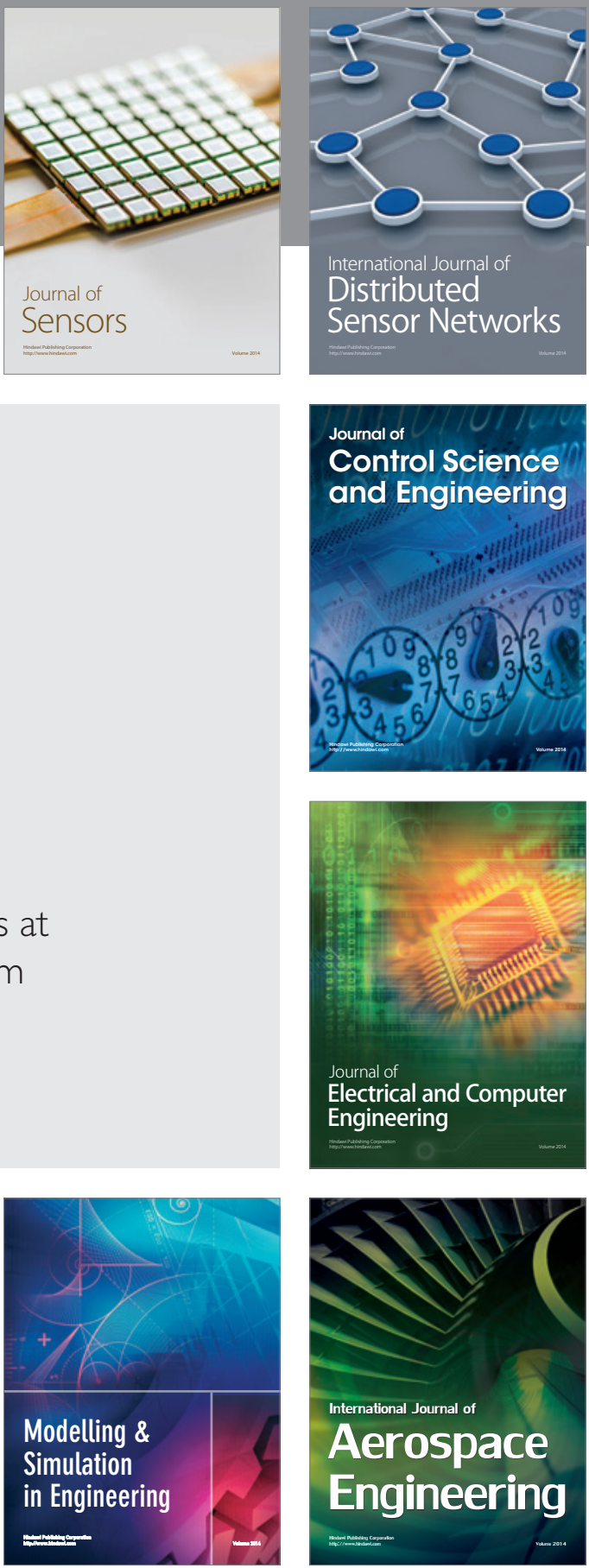

Journal of

Control Science

and Engineering
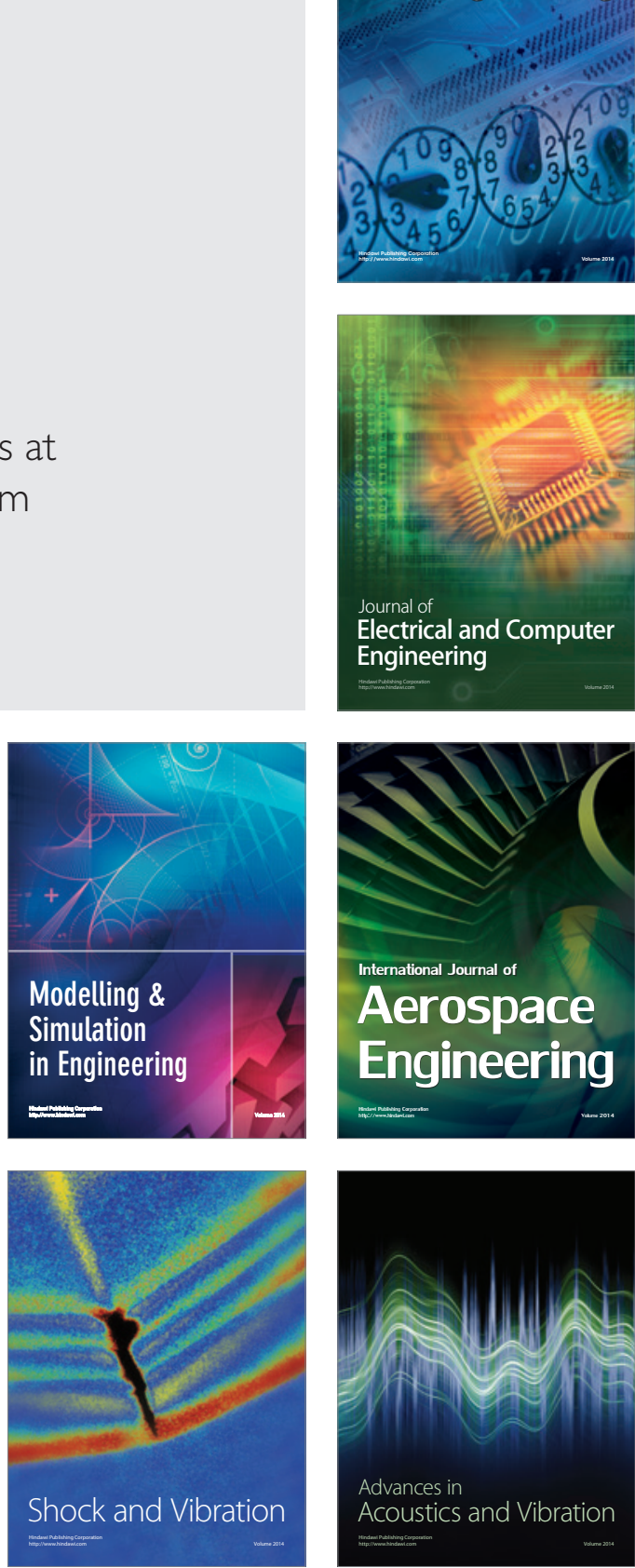